\title{
THE VASOINHIBIN SOLUTION STRUCTURE APPEARS UNFOLDED, DYNAMIC, AND FEATURES AGGREGATION
}

Jakob Triebel*1, Nicole Schauer ${ }^{1,2}$, Federico del Río Portilla³, Manuel Aguilar ${ }^{4}$, Juan-Pablo Robles ${ }^{4}$, Gonzalo Martínez de la Escalera ${ }^{4}$, Carmen Clapp ${ }^{4}$, and Thomas Bertsch ${ }^{1}$

${ }^{1}$ Institute for Clinical Chemistry, Laboratory Medicine and Transfusion Medicine, Paracelsus Medical University, Nuremberg, Germany ${ }^{2}$ Georg Simon Ohm University Nuremberg, Nuremberg, Germany

${ }^{3}$ Instituto de Química, Universidad Nacional Autónoma de México (UNAM), Ciudad Universitaria, Delegacíon Coyoacan, México D.F., México ${ }^{4}$ Instituto de Neurobiología, Universidad Nacional Autónoma de México (UNAM), Campus UNAM-Juriquilla, Querétaro, México

\section{BACKGROUND}

The pituitary hormone prolactin, the precursor of vasoinhibins, is structurally classified as a class-I helical cytokine with a four-helix bundle core and only a minimal degree of dark regions. Experimental data on the solution structure of vasoinhibins are not available.

\section{METHODS}

A recombinant, human vasoinhibin with a molecular mass of $16.7 \mathrm{kDa}$, comprising amino acids 29-176 of prolactin, was expressed in E. coli and purified. Nuclear magnetic resonance (NMR) spectra were obtained (1H 1D, 2D TOCSY, 500/700 Mhz, and 2D NOESY, $950 \mathrm{Mhz}$ ). The purified vasoinhibin sample was subjected to sodium dodecyl sulfate polyacrylamide gel electrophoresis and Western Blotting was performed using polyclonal and monoclonal, epitope-mapped anti-prolactin and anti-vasoinhibin antibodies.

\section{RESULTS}

1D NMR spectra at several conditions of $\mathrm{pH}$, buffer and temperature demonstrated broad vasoinhibin signals in a very narrow region with small differences between all spectra. Titration with trifluoroethanol and lithium chloride had minimal effect. TOCSY and NOESY experiments corroborated the results of all 1D experiments. Sodium dodecyl sulfate polyacrylamide gel electrophoresis and Western Blotting analyses demonstrated an anti-vasoinhibin antibody immunoreactive band with an apparent molecular mass of $16 \mathrm{kDa}$, consistent with the presence of the recombinant vasoinhibin-protein. Further immunoreactive bands of unknown identity with molecular masses of 28 and $35 \mathrm{kDa}$ were detected.
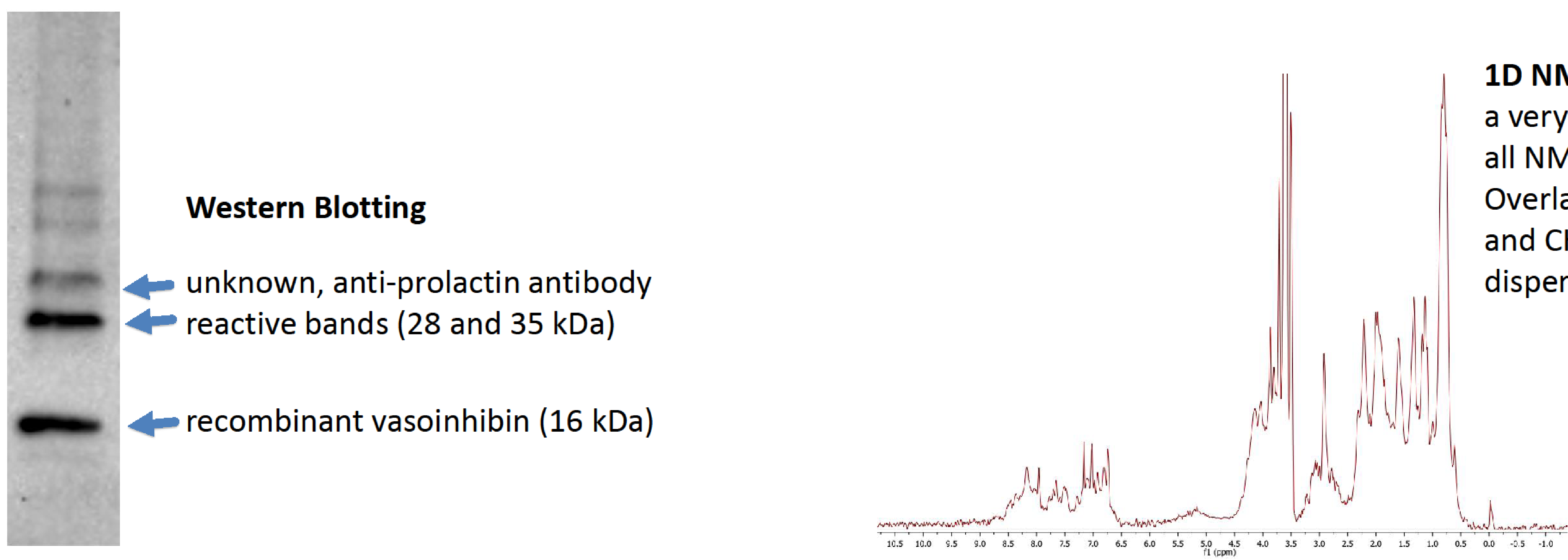

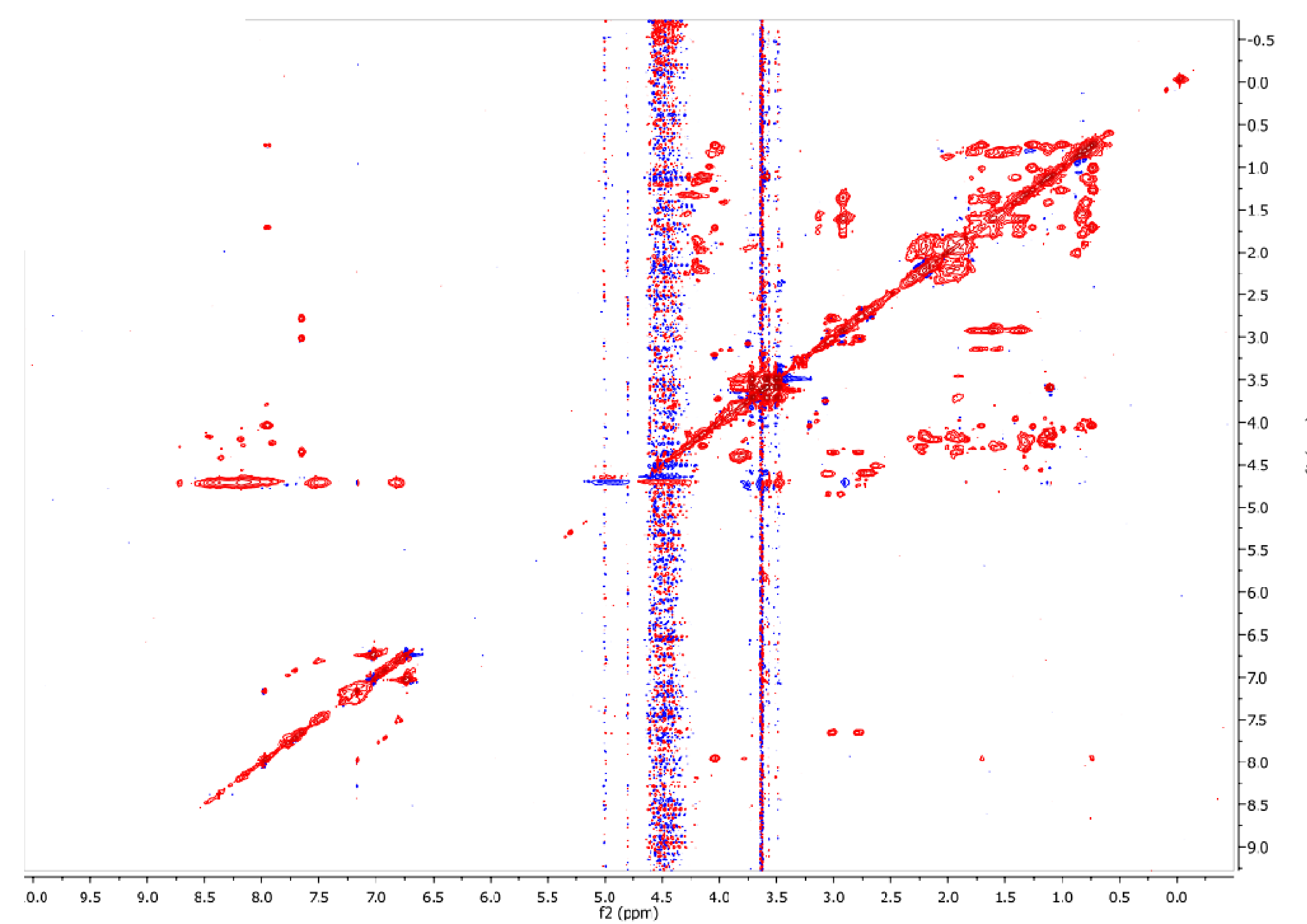

NMR TOCSY experiment shows reasonable cross signals in the aliphatic region. $\mathrm{NH}$ region lacks of enough correlations.
1D NMR Experiment shows a very narrow region where all NMR signals appear. Overlap is really crowded and $\mathrm{CH} 3$ groups are not disperse. 